\title{
An analysis of ${ }^{25} \mathrm{Al}$ energy levels observed in the ${ }^{28} \mathrm{Si}(\mathrm{p}, \alpha){ }^{25} \mathrm{Al}$ reaction
}

\section{S.T. Pittman ${ }^{1}$}

Department of Physics and Astronomy, University of Tennessee, Knoxville, TN 37996

E-mail: spittma1@utk.edu

\section{D.W. Bardayan}

Physics Division, Oak Ridge National Laboratory, Oak Ridge, TN 37831

E-mail: bardayandw@ornl.gov

\section{J.C. Blackmon}

Department of Physics and Astronomy, Louisiana State University, Baton Rouge, LA 70803

E-mail: blackmon@lsu.edu

\section{R.L. Kozub}

Physics Department, Tennessee Technological University, Cookeville, TN 38505

E-mail: rkozub@tntech.edu

\section{M.S. Smith}

Physics Division, Oak Ridge National Laboratory, Oak Ridge, TN 37831

E-mail: smithms@ornl.gov

The level structure of ${ }^{25} \mathrm{Al}$ has been studied at the ORNL Holifield Radioactive Ion Beam Facility (HRIBF) by measuring the angular and energy distributions of alpha particles from the ${ }^{28} \mathrm{Si}(\mathrm{p}, \alpha){ }^{25} \mathrm{Al}$ reaction. Proton beams $(\sim 10 \mathrm{nA})$ at laboratory energies of 40 - and $42-\mathrm{MeV}$ were generated by the $25 \mathrm{MV}$ tandem accelerator and bombarded a natural silicon target $\left(50 \mu \mathrm{g} / \mathrm{cm}^{2}\right)$. Alpha particles were detected and identified in the Silicon Detector Array (SIDAR) in the "telescope" configuration [1]. Eighteen levels have been observed and spins for several have been constrained through a distorted-wave Born approximation (DWBA) analysis of the angular distributions.

10th Symposium on Nuclei in the Cosmos

Mackinac Island, Michigan, USA

27 July - 1 August, 2008

\footnotetext{
$1 \quad$ Speaker
} 
The ${ }^{24} \operatorname{Mg}(\mathrm{p}, \gamma)^{25} \mathrm{Al}$ reaction occurs in many hot astrophysical environments, and the rate depends on the properties of ${ }^{25} \mathrm{Al}$ levels above the proton threshold $\left(\mathrm{E}_{\mathrm{x}}=2.271 \mathrm{MeV}\right)$. While the energy level diagram has been well-established for many low-lying ${ }^{25} \mathrm{Al}$ states, spin-parity assignments are missing or uncertain for several others. In particular, the levels at $\mathrm{E}_{\mathrm{x}}=1.613$, $3.424,4.026$, and $4.514 \mathrm{MeV}$ have uncertain spins, as tabulated in the latest compilation by Endt [4], and many levels above $4.9 \mathrm{MeV}$ have few constraints on the spins at all. The reaction ${ }^{28} \mathrm{Si}(\mathrm{p}, \mathrm{t}){ }^{26} \mathrm{Si}$ was previously studied at the ORNL Holifield Radioactive Ion Beam Facility (HRIBF) $[1,2,3]$. Here we report another reaction channel that was measured simultaneously, the ${ }^{28} \mathrm{Si}(\mathrm{p}, \alpha)^{25} \mathrm{Al}$ channel, and a determination of the ${ }^{25} \mathrm{Al}$ level structure. This paper represents the first spectroscopic analysis of the ${ }^{28} \mathrm{Si}(\mathrm{p}, \alpha)^{25} \mathrm{Al}$ reaction.

Proton beams at laboratory energies of $40-$ and $42-\mathrm{MeV}$ were generated by the $25 \mathrm{MV}$ tandem accelerator and bombarded a natural silicon target $\left(50 \mu \mathrm{g} / \mathrm{cm}^{2}\right)$. The beam current was $\sim 10 \mathrm{nA}$ and was integrated with a current integrator connected to a thick graphite beam stop. Alpha particles were detected and identified in the Silicon Detector Array (SIDAR) in the "telescope" configuration. This array is composed of 300- $\mu \mathrm{m}$-thick $(\Delta \mathrm{E})$ detectors backed by $500-\mu \mathrm{m}$-thick (E) detectors. Each detector consisted of 16 concentric strips, which covered laboratory angles $\Theta_{\mathrm{lab}}=18^{\circ}-48^{\circ}$ and $\Theta_{\mathrm{lab}}=31^{\circ}-75^{\circ}$ for the 40 - and $42-\mathrm{MeV}$ proton beams, respectively. The angles and solid angles subtended by the detector strips were calculated from the known detector geometry and checked with a calibrated ${ }^{244} \mathrm{Cm}$ alpha source. Alpha particles were detected and distinguished from other particles using standard energy-loss techniques.

Alpha energy spectra were compiled for alpha particles stopped in the E detectors as a function of strip number. Representative spectra are shown in Figure 1. Kinematic calculations at these angles revealed corresponding energy states of ${ }^{25} \mathrm{Al}$ produced in the reaction. The energy calibrations were internal, using documented levels of ${ }^{25} \mathrm{Al}$ from the compilation by Endt [4]. Values for observed ${ }^{25} \mathrm{Al}$ excitation energies in this study were calculated from an average of the value at each angle. The energy resolution was $\Delta \mathrm{E}_{\mathrm{c} . \mathrm{m} .} \approx 90 \mathrm{keV}$ for the $40 \mathrm{-MeV}$ run and $\Delta \mathrm{E}_{\mathrm{c} . \mathrm{m} .} \approx 145 \mathrm{keV}$ for the $42-\mathrm{MeV}$ run, where kinematical broadening contributed to the loss of energy resolution at larger detection angles. For this reason, excitation energies were extracted from the $40-\mathrm{MeV}$ data when possible.

Angular distributions for strongly-populated levels were analyzed with the zero-range distorted wave Born approximation (DWBA) code DWUCK-IV of Kunz [5] using the triton cluster form factor for the transferred nucleons. Alpha optical and triton cluster parameters were adopted from Hoyler et al. [6], who studied the similar-mass reaction ${ }^{27} \mathrm{Al}(\mathrm{p}, \alpha)^{24} \mathrm{Mg}$. Small modifications of the triton cluster parameters were required to better fit the angular distributions for known levels. In particular, values were adjusted for the reduced radius and diffuseness of the real potential to better fit the experimental data. Proton parameters were derived from Abdel-kariem [7], which originated with Snelgrove and Kashy [8], and were supplemented with values from the DWUCK-IV test case [5].

The angular momentum transfer was determined from the spins of the ${ }^{28} \mathrm{Si}$ ground state, the triton cluster, and the ${ }^{25} \mathrm{Al}$ daughter state to be evaluated. To achieve the correct spin of the ${ }^{25} \mathrm{Al}$ ground state $\left(5 / 2^{+}\right)$, for example, the $0^{+}$ground state of ${ }^{28} \mathrm{Si}$ must eject the $1 / 2^{+}$triton with a transfer angular momentum of $\mathrm{L}=2$. 


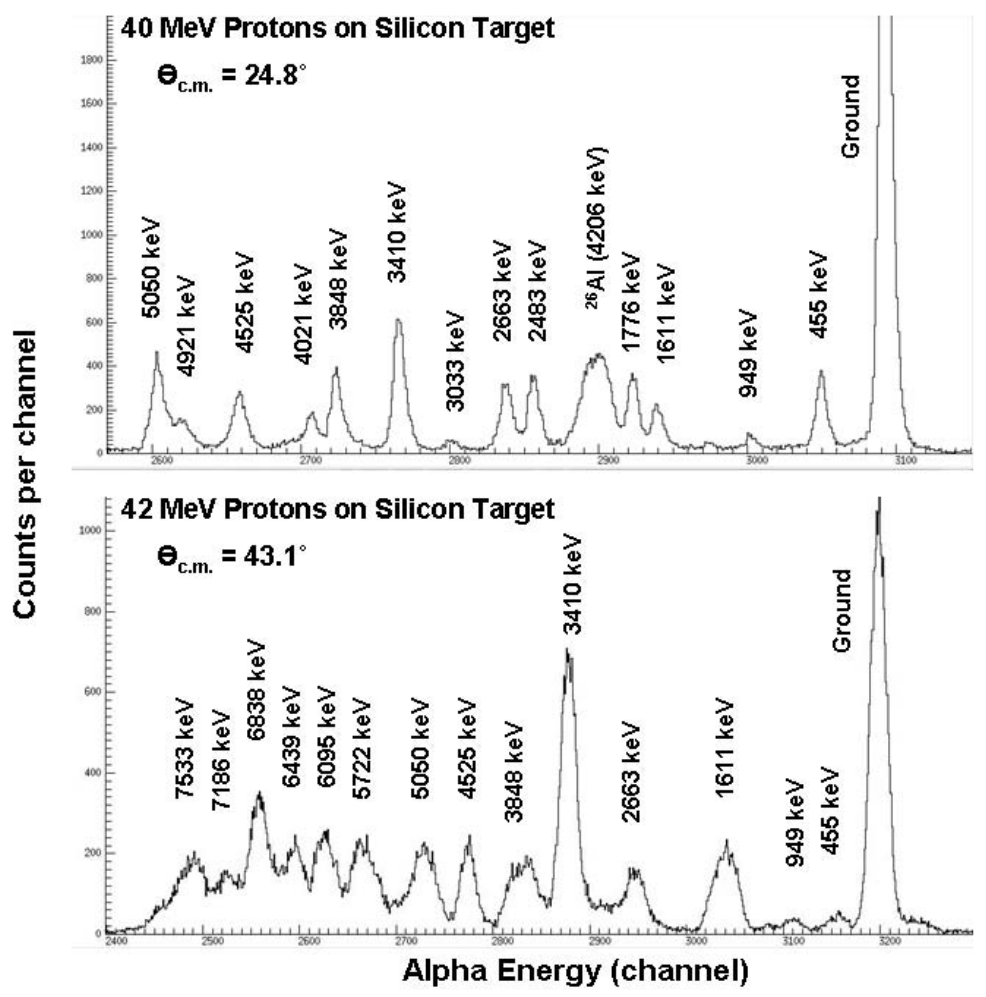

Figure 1. Representative alpha spectra for the $40-$ and $42-\mathrm{MeV}$ runs are shown in the top and bottom plots, respectively.

The number of nodes in the triton cluster wave function was determined by the "harmonic oscillator energy" conservation rule, for 1s internal motion of the cluster [9]. It is assumed that the triton cluster is bound in a Wood-Saxon potential well with a binding energy equal to its separation energy and that the number of nodes and momentum of this wave function are limited by the Talmi-Moshinsky relation $2 \mathrm{~N}+\mathrm{L}=\mathrm{Q}=\Sigma \mathrm{q}_{\mathrm{i}}$, where $\mathrm{Q}$ is the sum of oscillator quanta for the transferred triton [6,7]. A triton of even external momentum transferred from ${ }^{28} \mathrm{Si}$ would likely originate from the $1 \mathrm{~d}_{5 / 2}$ shell of the $2 \mathrm{~s} 1 \mathrm{~d}$ shell, and require a value of $\mathrm{Q}=6$, as each nucleon has an oscillator quantum $\mathrm{q}=2$. This results in a positive-parity ${ }^{25} \mathrm{Al}$ state. To achieve a negative-parity state of ${ }^{25} \mathrm{Al}$, the triton would have to consist of 2 nucleons from the $2 \mathrm{~s} 1 \mathrm{~d}$ shell, as well as a single nucleon from the $1 \mathrm{p}$ shell. Such a situation demands $\mathrm{Q}=5$ in the Talmi-Moshinsky transformation. The two nucleons from the $2 \mathrm{~s} 1 \mathrm{~d}$ shell have $\mathrm{q}=2$, while the one from the $1 \mathrm{p}$ shell has $\mathrm{q}=1$.

Differential cross-sections of ${ }^{25} \mathrm{Al}$ states were plotted with DWBA predictions to confirm existing spin-parity designations for known states in ${ }^{25} \mathrm{Al}$ and to suggest clarification of and classification for uncertain or previously-unknown states. These plots are shown in Figure 2. Gaps appear in the angular distributions where the peak of interest was obscured by a contaminant peak from ${ }^{29} \mathrm{Si}(\mathrm{p}, \alpha)^{26} \mathrm{Al}$ or ${ }^{12} \mathrm{C}(\mathrm{p}, \alpha){ }^{9} \mathrm{~B}$, or if alpha particles populating the state did 
not stop in the E detector. While the analysis is not yet complete, it appears that constraints on spins will be obtained for several levels.

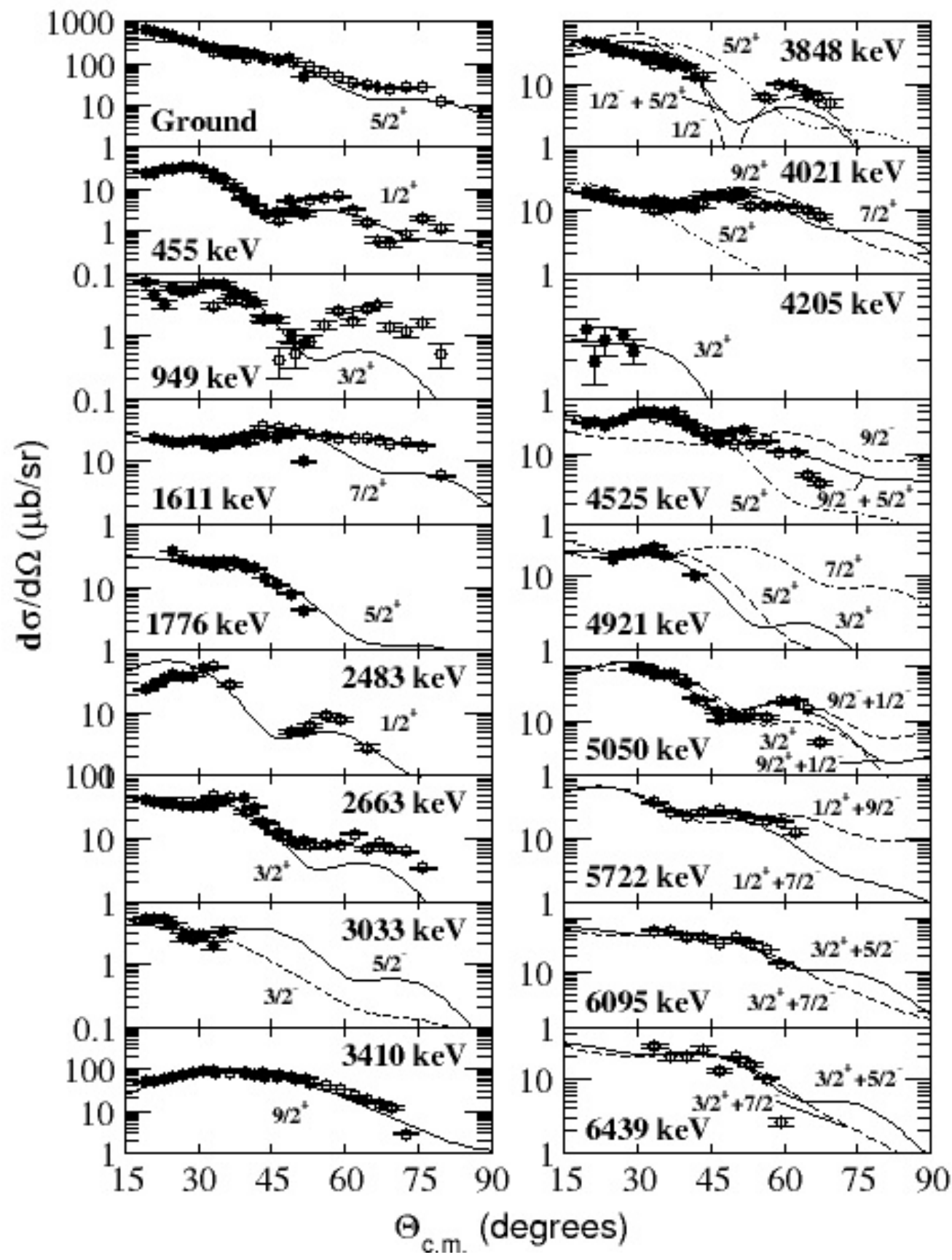

Figure 2. Differential cross-sections for the ${ }^{28} \mathrm{Si}(\mathrm{p}, \alpha)^{25} \mathrm{Al}$ reaction along with DWBA fits.

A study of the ${ }^{28} \mathrm{Si}(\mathrm{p}, \alpha)^{25} \mathrm{Al}$ reaction at the Oak Ridge HRIBF facility has suggested spectroscopic identities for some of the ${ }^{25} \mathrm{Al}$ excited states. Analysis is ongoing and will be 
presented in a future report. Further research will have to be done on this nucleus, as details of its energy spectrum are crucial to the calculation of the ${ }^{24} \mathrm{Mg}(\mathrm{p}, \gamma){ }^{25} \mathrm{Al}$ reaction rate in stellar environments.

This work was supported by the U. S. Department of Energy under contract numbers DE-FG02-96ER40983 (UTK), DE-AC05-00OR22725 (ORNL), and DE-FG02-96ER40995 (TTU).

\section{References}

[1] D.W. Bardayan, J.C. Blackmon, A.E. Champagne, A.K. Dummer, T. Davinson, U. Greife, D. Hill, C. Iliadis, B.A. Johnson, R.L. Kozub, C.S. Lee, M.S. Smith, and P.J. Woods, Phys. Rev. C 65, 032801 (2002).

[2] D.W. Bardayan, J.C. Blackmon, A.E. Champagne, A.K. Dummer, T. Davinson, U. Greife, D. Hill, C. Iliadis, B.A. Johnson, R.L. Kozub, C.S. Lee, M.S. Smith, and P.J. Woods, Nuc. Phys. A 718, 505c (2003).

[3] D.W. Bardayan, J.A. Howard, J.C. Blackmon, C.R. Brune, K.Y. Chae, W.R. Hix, M.S. Johnson, K.L. Jones, R.L. Kozub, J.F. Liang, E.J. Lingerfelt, R.J. Livesay, S.D. Pain, J.P. Scott, M.S. Smith, J.S. Thomas, and D.W. Visser, Phys. Rev. C 74, 045804 (2006).

[4] P.M. Endt, Nuc. Phys. A 633, 1 (1998).

[5] P.D. Kunz, Computer Code DWUCK-IV. University of Colorado, Boulder (1978).

[6] F. Hoyler, H. Oberhummer, T. Rohwer, and G. Staudt, Phys. Rev. C 31, 17 (1985).

[7] S.E. Abdel-kariem, Appl. Rad. Isot. 64, 925 (2006).

[8] J.L. Snelgrove and E. Kashy, Phys. Rev. 187, 1259 (1969).

[9] F. Pellegrini, G. Calvelli, P. Guazzoni, and S. Micheletti, Phys. Rev. C 18, 613 (1978). 